\title{
Alternative methods to determine the elastoplastic properties of sintered hydroxyapatite from nanoindentation testing
}

\author{
S. Naderi ${ }^{\text {a,c,*, }}$ M.A. Hassan ${ }^{\text {a,b,c,*, }}$ A.R. Bushroa ${ }^{a, c}$ \\ ${ }^{a}$ Mechanical Engineering Department, Faculty of Engineering, University of Malaya, 50603 Kuala Lumpur, Malaysia \\ ${ }^{\mathrm{b}}$ Mechanical Engineering Department, Faculty of Engineering, Assiut University, 71516 Assiut, Egypt \\ 'Center of Advanced Manufacturing and Material Processing, AMMP Center, University Malaya, 50603 Kuala Lumpur, Malaysia
}

A R T I C L E I N F O

\section{Article history:}

Received 8 September 2014

Accepted 21 November 2014

Available online 27 November 2014

\section{Keywords:}

Hydroxyapatite

Nanoindentation

Mechanical properties

Finite element model

Inverse model

\begin{abstract}
A B S T R A C T
This study introduces alternative methods to determine the elastoplastic properties of bovine-derived Hydroxyapatite (HA) porous bone graft through a set of nanoindentation tests with a Berkovich indenter Generally, experimental data obtained from nanoindentation tests are force displacement, hardness and elastic modulus. However, to determine plastic properties such as strength coefficient and work hardening exponent of bovine HA, analytical or inverse finite element models are required. In this paper, the effect of sintering temperature on these properties of HA is studied for the range of $1000-1400^{\circ} \mathrm{C}$. The direct and inverse Finite Element (FE) simulation models for nanoindentation tests were written in MSC, MARC ${ }^{*}$ software. A special algorithm for the inverse technique was developed to infer the most suitable elastoplastic material model for HA. A semi-empirical method was adapted to calculate the elastoplastic material properties of HA. The numerical results of harder hydroxyapatite showed better agreement with the experiments while the work hardening exponent, or $n$-value, and strength coefficient $k$ of hard HA were found to be 0.23 and $8.05 \mathrm{GPa}$ respectively. A comparison between the experimental and predicted load-displacement curves showed that the proposed inverse technique is effective in predicting the elastoplastic material properties from the nanoindentation test with error below $4 \%$ at maximum load.
\end{abstract}

(c) 2014 Elsevier Ltd. All rights reserved.

\section{Introduction}

Hard tissue replacement materials and synthetic bone are widely considered owing to the lack of autograft materials and health risks posed by allograft. Hydroxyapatite is the dominan inorganic phase in natural bone. Synthetic hydroxyapatite particles, film, coating, fiber and porous skeleton are used broadly. HA has been extensively investigated recently due to its chemical and structural similarities to naturally occurring biological apatite in hard tissues. The main weakness of HA is that its mechanical properties are not as good as bone tissue, particularly the plastic behavior. Nonetheless, HA has a much higher elastic modulus ( $120 \mathrm{GPa}$ [1]) than bone ( $<30 \mathrm{GPa}$ for trabecular bone [2]). Besides, HA is known as a brittle material while bone shows significant plastic behavior $[3,4]$. The large differences in elastic modulus

* Corresponding authors at: Mechanical Engineering Department, Faculty of Engineering, University of Malaya, 50603 Kuala Lumpur, Malaysia. Tel.: +60 17 3421068; fax: +60 379677669 (S. Naderi). Tel.: +60 3 79674447; fax: +60 3 79675282 (M.A. Hassan)

E-mail addresses: sadjad.naderi@siswa.um.edu.my (S. Naderi), mohsenegypt @um.edu.my (M.A. Hassan).

http://dx.doi.org/10.1016/j.matdes.2014.11.030

0261-3069/○ 2014 Elsevier Ltd. All rights reserved. between $\mathrm{HA}$ and bone, as well as lack of plastic ability of HA result in greater stress concentration and fracture at the bone-material interface. Furthermore, from a material properties point of view, the elastoplastic behavior of HA remains unknown, including various hardening parameters and how to identify them. These factors define the mechanical behavior of HA and they are important to a material for bone substitution.

A general study has been performed on the mechanical properties of HA, including elastic modulus, hardness and strength. Abinitio technique was implemented to calculate the elastic constant and carry out a theoretical tensile experiment on stoichiometric hydroxyapatite crystal [5]. The deformation behavior was studied using structural analysis under various levels of tensile strain. Several attempts have been made to find the connection between porosity, elastic properties and fracture strength of sintered HA [6,7]. To the best of our knowledge, however, proper micromechanical analysis of the effective elastoplastic properties has never been done; the shape of the pores was either unnoticed or assumed to be ideally spherical [6]. A research was performed on the role of microstructure and porosity on the mechanical behavior of sintered hydroxyapatite [8]. Also the effect of cell number, size and 
density on the mechanical behavior of polymer foam has been studied [9]. Work was only done experimentally to observe the porous influence and it was concluded that porous microstructure produces HA with plastic deformation properties. The dependence of the mechanical properties of sintered hydroxyapatite on sintering temperature was discussed [10]. The problem was clarified by concentrating on the elastic modulus, compression strength and micro structure of sintered hydroxyapatite on processing temperature. However, the previous work has been done on hardening parameters for alloy [11] but for HA the identification of elastic and plastic parameters remains unsolved. It is useful to consider sintering process of the powder base material to determine the influence over mechanical properties [12]. Generally, research has focused on elastic properties; consequently, the plastic area and actual microstructure of sintered HA is still unknown.

Compared with mechanical properties tests, the nanoindentation technique is an appropriate means of characterizing nanomechanical properties. A number of analysis approaches based on experimental and analytical research have been directed at many materials to extract the mechanical properties from the nanoindentation load-displacement curve. During loading, the typical load-displacement response generally follows the relation described by Kick's law, that is, $P=C h^{2}$ with loading curvature $C$. Based on Kick's law, mechanical properties are obtained from measured force-displacement curves, such as elastic modulus, yield strength, strain hardening, residual stress and creep behavior [13-17]. In this study, an analysis method for HA is implemented to extract the hardening parameters from the measured data set of load and displacement for Berkovich indentation.

The main goal in present paper is to obtain accurate mechanical properties for bovine hydroxyapatite through different alternative methods. The mechanical behavior of HA is investigated using Berkovich nanoindentation test to obtain the hardening parameters. FE-inverse model besides an analytical method is presented to identify the nonlinear elastoplastic mechanical properties. These methods are validated with existing experimental data under sintering temperature that has a significant role on HA mechanical properties. The proposed methods save time and provide low-cost compared with the experimental-numerical technique. The predicted results are discussed and concluded.

\section{Materials and methods}

\subsection{HA powder preparation}

Initially, bovine samples were supplied from a local slaughter house. The macroscopic adhering impurities and material were removed from the cortical bone in order to clean away any ligaments and tissue from the sample. The acquired bones were boiled in distilled water, and they were then dried in the sun to eliminate organic matter and collagen. A defatting process was implemented so that soot would not form in the heating step. A hacksaw was used to cut the samples into small pieces of $10 \times 10 \times 5 \mathrm{~mm}$. The cut, rectangular bones were heated for $2 \mathrm{~h}$ in air at $900^{\circ} \mathrm{C}$ at a heating rate of $5{ }^{\circ} \mathrm{C} / \mathrm{min}$ to get rid of the organic matrix. With slow furnace cooling the samples returned to room temperature. A mortar pestle and sieve were employed to obtain HA powder. In this paper, the particle size considered was below $400 \mu \mathrm{m}$ for the HA powder.

\subsection{HA powder characterization}

$\mathrm{X}$-ray diffraction with a monochromated $\mathrm{Cu} \mathrm{K} \alpha$ radiation model XRD 6000 Shimadzu was used to analyze the hydroxyapatite phases. Scanning speed and step were adjusted to $7 \% \mathrm{~min}$ and $0.02^{\circ}$ respectively. The Fourier transform infrared (FTIR) spectrum of the powder was identified with a Nicolet Avatar 360 FTIR spectrometer. The powder and $\mathrm{KBr}$ were mixed before FTIR analysis, and then they were pressed into pellets. During these processes, a scanning electron microscope (SEM: Hitachi S4200B) was used to test the bone samples and HA powder morphology. The samples were mounted on steel stub pins and afterward coated with palladium (Pd) using a sputter coater (E1030 Ion Sputter, Hitachi) in order to make a conducting layer and avoid charging in the microscope. The screening method (sieve shaker machine, Retsch AS200) was chosen to analyze the distribution of bovine HA powder particles. Screens were stacked starting with $20 \mu \mathrm{m}$, to $45 \mu \mathrm{m}, 150 \mu \mathrm{m}$ and $300 \mu \mathrm{m}$ for screening analysis (the smallest opening size was placed at the bottom). $300 \mathrm{gr}$ of the powder was placed on top of the screen stack, which was vibrated for $60 \mathrm{~min}$. Subsequent to vibration, the powder in each size space was weighed and the interval percent was estimated for every size fraction. The particle size data points were mapped on a particle size distribution histogram.

\subsection{Sample preparation}

Using $20 \mathrm{~mm}$ cylindrical dies, the HA powder was compacted uniaxially at $156 \mathrm{MPa}$ to form green bodies, which were then sintered in air atmosphere with no pressure at different temperatures, $1000,1100,1200,1300$ and $1400{ }^{\circ} \mathrm{C}$, at a furnace ramp rate of $5{ }^{\circ} \mathrm{C} /$ min and $2 \mathrm{~h}$ dwelling time. The disk-shaped HA samples were $3 \mathrm{~mm}$ thick and $10 \mathrm{~mm}$ in diameter.

\subsection{Nanoindentation test}

Indentation experiments were performed with a nanoindentation machine (Triboscope system, Hysitron Inc., USA). ISO 145774 [18] standard was followed for the nanoindentation test. A calibrated Berkovich indenter was used with included angle $(\alpha)$ $65.03^{\circ}$ and radius of tip curvature of $150 \mathrm{~nm}$, as shown in Fig. 1. Diamond was used for the indenter with elastic modulus of $1140 \mathrm{GPa}$ and Poisson's ratio of 0.07 . The maximum indentation depths at which material properties become almost constant in the nanoindentation test were obtained with different maximum loads for different samples. For the HA samples at $1100-1400{ }^{\circ} \mathrm{C}$ sintering temperatures, the maximum indentation load was equal

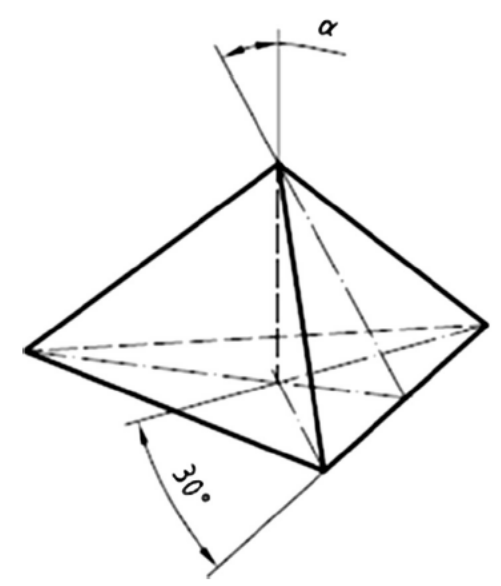

Fig. 1. Geometry of Berkovich indenter with $65.3^{\circ}$ angle according to ISO 14577 


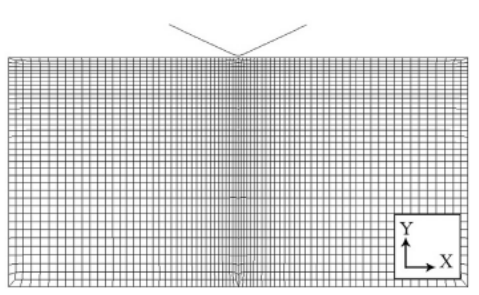

(a)

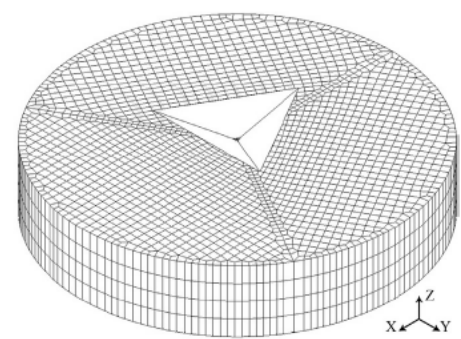

(b)

Fig. 2. Finite element mesh for the Berkovich indentation test: (a) 2D model, (b) 3D model.

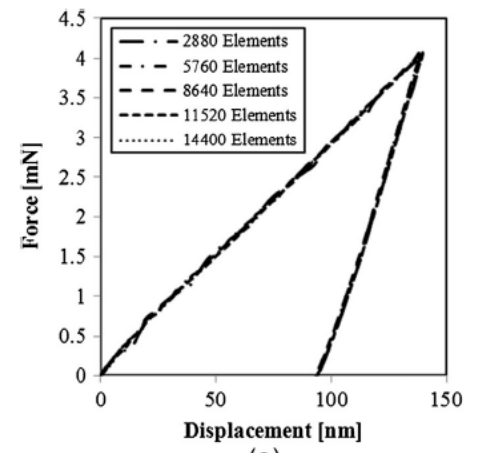

(a)

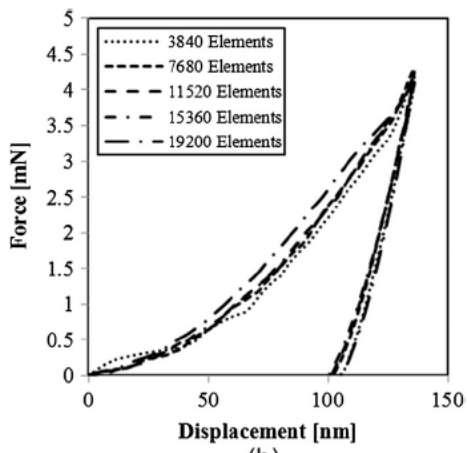

(b)

Fig. 3. Effect of mesh size on the force-displacement curves obtained by incremental displacement solution method for: (a) 2D model, and (b) 3D model.

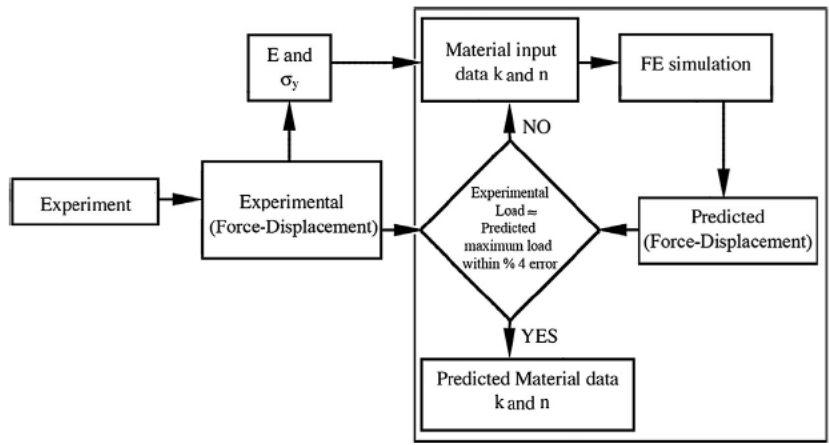

Fig. 4. Outline of the inverse FE technique to determine the plastic properties of $\mathrm{HA}$ from nanoindentation experimental data.

to $4 \mathrm{mN}$ to reach a maximum indentation depth of $150 \mathrm{~nm}$. For the HA sample sintered at $1000^{\circ} \mathrm{C}$, this depth was obtained by applying a load of $1.5 \mathrm{mN}$. At least five indentations were made at each load level on each sample. Poisson's ratio can be assumed a constant value of 0.2 in numerical analysis for ceramics [19]. The indentation force was applied for 30,10 and 30 s for loading, holding and unloading respectively, with a constant speed.

\section{Semi-empirical method}

Extensive analyses of indentation data obtained with a wedgetype indenter revealed that for incompressible materials, the overall relationship between the hardness and yield stress $\sigma_{y}$ of an elastoplastic material can be expressed as [20]:

$R_{y}=\frac{H}{\sigma_{y}}=\frac{\xi}{\sqrt{3}}\left[1+\ln \left(\frac{4 \sigma_{y}}{3 \pi E} \tan \beta\right)\right]$

where $E$ is Young's modulus of the material, and $\xi$ is a parameter which depends on the ratio of the yield stress and Young's modulus of the intended material. For hard metallic alloys and ceramics, $\xi$ may be selected as $5 \sqrt{2} / 9$ and 1 , respectively.

Material hardness is defined as the ratio between the applied force and the projected contact area. The actual projected contact 


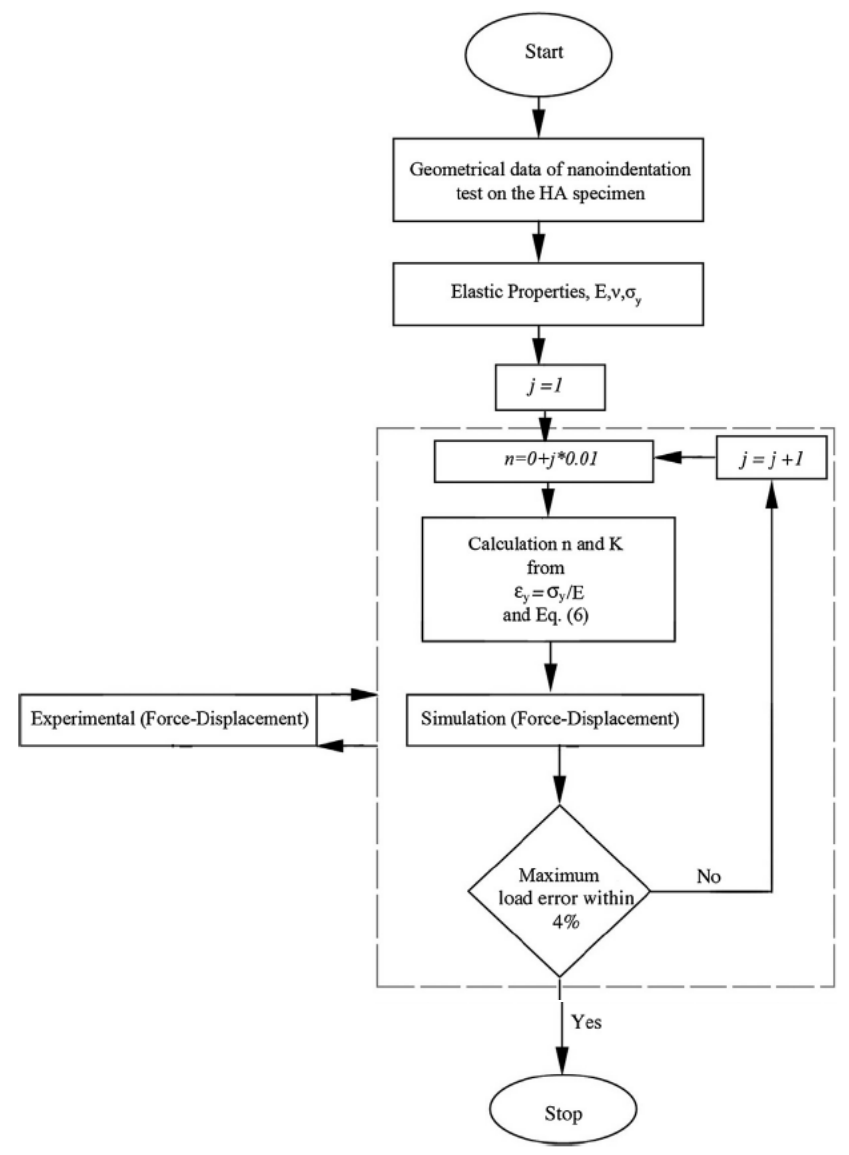

Fig. 5. Procedure to determine the hardening parameters through a force displacement curve using nanoindentation data and inverse finite element technique.

Table 1

Work hardening parameters of hydroxyapatite at different sintering temperatures as calculated from the semi-empirical model.

\begin{tabular}{|c|c|c|c|c|c|c|c|c|c|c|c|c|}
\hline No. & Sintering temperature $\left({ }^{\circ} \mathrm{C}\right)$ & $E(\mathrm{GPa})$ & $H(\mathrm{GPa})$ & $\sigma_{y}(\mathrm{GPa})$ & $A_{m}\left(\mathrm{~nm}^{2}\right)$ & $S_{m}(\mu \mathrm{N})$ & $\bar{E}(\mathrm{GPa})$ & $C(\mathrm{~nm})$ & $\sigma_{0.29}(\mathrm{GPa})$ & $\varepsilon_{y}$ & $n$ & $K(\mathrm{GPa})$ \\
\hline 1 & HA- 1000 & 47.4 & 1.51 & 0.69 & $1.21 \mathrm{E} 6$ & 64.353 & 47.3 & 0.0026 & 19.296 & 0.014 & 0.46 & 4.92 \\
\hline 2 & HA- 1100 & 77.6 & 2.97 & 1.46 & $1.97 \mathrm{E} 6$ & 100.59 & 57.9 & 0.0651 & 23.726 & 0.018 & 0.39 & 6.99 \\
\hline 3 & HA- 1200 & 150.3 & 6.46 & 3.35 & $6.32 \mathrm{E} 5$ & 119.16 & 38.3 & 0.0897 & 15.606 & 0.022 & 0.21 & 7.80 \\
\hline 5 & HA- 1400 & 87.2 & 4.29 & 2.36 & $9.23 \mathrm{E} 5$ & 85.79 & 22.8 & 0.0936 & 9.154 & 0.027 & 0.30 & 6.97 \\
\hline
\end{tabular}

Table 2

Hardening parameters determined by the inverse technique for 2D and 3D models.

\begin{tabular}{lrlllll}
\hline \multicolumn{2}{l}{ Sintering temperature $\left({ }^{\circ} \mathrm{C}\right)$} & 1000 & 1100 & 1200 & 1300 & 1400 \\
\hline$n$ & 2D-model & 0.43 & 0.39 & 0.23 & 0.19 & 0.31 \\
& 3D-model & 0.40 & 0.35 & 0.23 & 0.19 & 0.31 \\
$K(\mathrm{GPa})$ & 2D-model & 4.32 & 6.99 & 8.06 & 7.26 & 7.23 \\
& 3D-model & 3.80 & 5.96 & 8.06 & 7.26 & 7.23
\end{tabular}

area $A_{m}$ can be computed from the hardness value $H$ and the maximum load of indentation $P_{m}$ using Eq. (2).

$H=P_{a v e}=\frac{P_{m}}{\Lambda_{m}}$

Reduced elastic modulus $\bar{E}$ can be obtained based on $\Lambda_{m}$ and the slope of the unloading curve at maximum force and maximum depth $\left(S_{m}=\left.\frac{d P}{d h}\right|_{h=h_{m}}\right)[21]$ :

$\bar{E}=\frac{S_{m}}{c^{*} \sqrt{\Lambda_{m}}}$

where $c^{*}$ is a computationally driven parameter that depends on the geometry of the indenter and its value for different indentation tests can be used to find the reduced elastic modulus for the indented material. For a Berkovich indenter, the $c^{*}$ value is 1.2370 .

For most materials, the curvature $C$ may be expressed as a function of the characteristic stress $\sigma_{0.29} \bar{E}$ and $\sigma_{y}$ [22-24] as formulated in Eq. (4): 
$C=\frac{P}{h^{2}}=A \sigma_{0.29}\left\{1+\frac{\sigma_{y}}{\sigma_{0.29}}\right\}\left\{B+\ln \left(\frac{\bar{E}}{\sigma_{y}}\right)\right\}$

where $A$ and $B$ are constant and depend on the indenter geometry. The values of $A$ and $B$ are 6.02 and -0.875 respectively for a Berkovich indenter. $\sigma_{029}$ is the stress which corresponds to the characteristic strain $\varepsilon_{r}=0.29$ for the indented material under uniaxial compression. The characteristic strain is an important parameter in such analysis and should be carefully selected. Pervious FEM analyses for sharp indenters such as Berkovich and Vickers indenters suggests that $\varepsilon_{r}=0.29$ [22].

Simply, yield strain is calculated from $\varepsilon_{y}=\sigma_{y} / E$; consequently, the work hardening exponent can be computed from Eq. (5) using $\sigma_{0.29}, \sigma_{y}$ and $\varepsilon_{y}$ :

$n=\frac{\ln \left(\sigma_{0.29}\right)-\ln \left(\sigma_{y}\right)}{\ln \left(\varepsilon_{0.29}\right)-\ln \left(\varepsilon_{y}\right)}$

The strength coefficient of the material is obtained using Eq. (6). $\sigma_{y}=K \varepsilon_{y}^{n}$

\section{Direct FE model}

The direct finite element model was made using MSC.Marc software. Fig. 2 illustrates the two dimensional (2D) and three dimensional (3D) mesh designs of the entire model with a magnified view of the mesh design under a Berkovich indenter. In the 3D simulation, the HA sample was modeled into a cylindrical shape

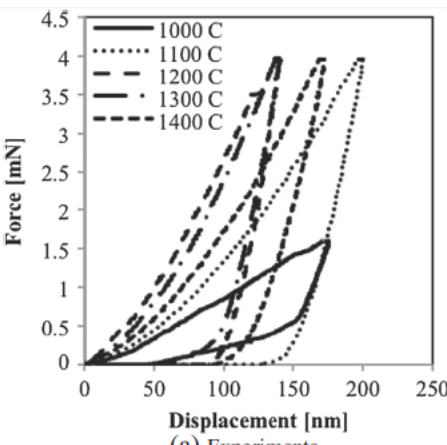

(a) Experiments

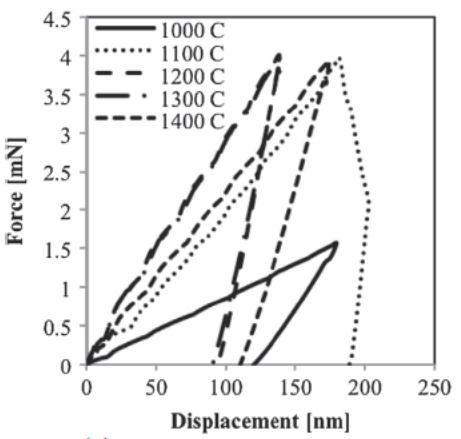

(c) 2D model (Displacement- Control) rather than cubic in order to obtain proper mesh design in three radial directions. In the nanoindentation simulation, the indented surface is a free surface; the shape does not significantly affect stress distribution if the surface is considered large enough. The indenter and HA were considered rigid and elastoplastic materials, respectively. The mechanical properties of hydroxyapatite were calculated based on the analytical method and experimental data. These HA properties were used in the simulation to obtain nearly the same force-penetration depth as the experimental nanoindentation test results.

In this study, the numerical analysis steps include only loading and unloading but not holding since the strain rate is not reflected in the material properties. Two methods were chosen to obtain a displacement-load curve from the FE model, which are force control and displacement control. Force control was adjusted according to the indentation force applied in the experiments; the maximum depth of indentation was implemented in the displacement control method. In the 3D model, only displacement control was used.

As the nanoindentation test was assumed to be quasi-static, the strain rate sensitivity was not considered. The adopted meshes are 4-node plain stress quads and 8-node 3D solid elements for 2D and $3 \mathrm{D}$ respectively. The contact between the indenter and the sample surface was modeled as rigid to deformable. Throughout the analysis, the nodes of the back face of the sample were constrained against displacement in the $x$ - and $y$-directions for 2D and plus $z$-direction for 3D. The total deformation was calculated by the axial component of nodal displacement in the load direction for the node touching the indenter tip.

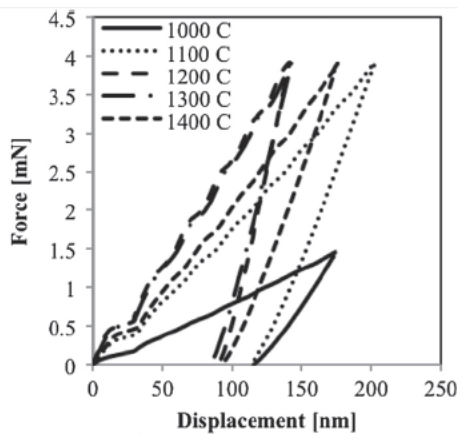

(b) 2D model (Force - Control)

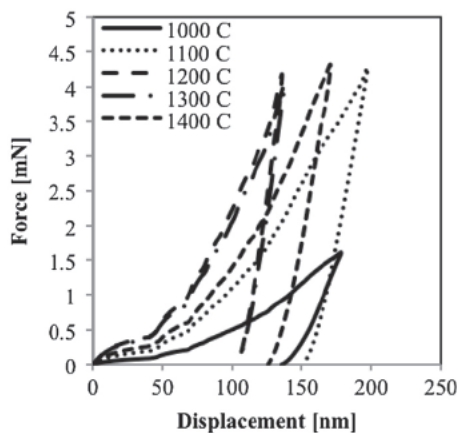

(d) 3D model

Fig. 6. Force displacement curves obtained from the nanoindentation test of $\mathrm{HA}$ and finite element analysis; material parameters are based on Table 1 for different sintering temperatures, $1000,1100,1200,1300$ and $1400^{\circ} \mathrm{C}$ in $2 \mathrm{D}$ and 3D.

\section{Link to Full-Text Articles :}

\section{http://www.sciencedirect.com/science/article/pii/S0261306914009510}

